\title{
Analyse des structures planaires multicouches à ferrite par la méthode des éléments finis
}

\author{
M. Meliani *, M. Feham, B. Benbakhti \\ Université de Tlemcen, Département d'Electronique, B.P. 230 Chetovane, 13000 Tlemcen, \\ Algérie
}

(Reçu le 14 Avril 2005, accepté le 25 Août 2005)

*Correspondance et tirés à part, e-mail : fehmag@yahoo.fr

\section{Résumé}

L'analyse électromagnétique des circuits intégrés micro-ondes est un sujet attrayant dô au développement récent et l'utilisation de nouveaux matériaux tels que les matériaux anisotropes magnétiques (hexaferrite, ...). Ces matériaux sont employés dans beaucoup de composants micro-ondes (isolateurs, circulateurs, déphaseurs) utilisés dans des systèmes de télécommunication. La conception de ces dispositifs exige une caractérisation électromagnétique rigoureuse de leurs performances. Cet article présente les résultats d'analyse par la méthode des éléments finis de plusieurs structures planaires multicouches diélectrique et ferrite polarisé longitudinalement selon la direction de propagation. Cette méthode permet la détermination de la perméabilité effective, de l'impédance caractéristique, des paramètres primaires du circuit électronique équivalent des structures planaires propageant le mode fondamental quasiTEM. L'influence de la fréquence et de l'intensité de l'aimantation $H_{0}$ du ferrite sur ces paramètres devient alors possible avec cette méthode.

Mots-clés : Déphaseurs planaires, coupleurs micro-ondes, substrat à ferrite, mode quasi-TEM, caractérisation électromagnétique, méthode des éléments finis

\section{Abstract}

\section{Finite element analysis of multi-layer planar structures with Ferrite}

Electromagnetic Analysis of microwave integrated circuits is an attractive subject do to the recent development and use of new materials such as magnetic anisotropic materials (hexaferrite, ...). These materials are used in many microwave components (isolator, 
circulator, phase shifter) employed in telecommunication systems. The design of these devices requires a rigorous electromagnetic characterization of their performances. This paper presents the analysis by the finite element method of several planar structures with dielectric and ferrite layers polarized longitudinally in the direction of propagation. This method allows the determination of the effective permeability, the characteristic impedance, the primary parameters of the equivalent circuit of planar structures propagating the quasi-TEM mode. The influence of the frequency and the magnetization $H_{0}$ on these parameters is then derived.

Keywords : Planar phase shifters, microwave couplers, ferrite substrate, quasi-TEM mode, electromagnetic characterization, finite element method.

\section{Introduction}

Les matériaux gyromagnétiques ont été largement répandus dans des circuits microondes tels que les isolateurs, les circulateurs et les déphaseurs.

Récemment, plusieurs auteurs se sont intéressés à la caractérisation de matériaux magnétiques en couche ou sous forme de film [1-5]. Cet article présente les résultats de la caractérisation électromagnétique de plusieurs structures micro-ondes composées de lignes de transmission suspendues sur une couche de ferrite polarisée longitudinalement (selon la direction de propagation).

Dans ce cas, la perméabilité du ferrite est exprimée par le tenseur de Polder :

$$
\bar{\mu}=\left[\begin{array}{ccc}
\mu & -j k & 0 \\
+j k & \mu & 0 \\
0 & 0 & 1
\end{array}\right] \quad \mu_{0}=\mu_{r} \mu_{0}
$$

Les paramètres $\mu$ et $k$ sont définis par [2]:

$\mu=1+\frac{\left(\gamma \mu_{0}\right)^{2} M H_{0}}{\left(\gamma \mu_{0} H_{0}\right)^{2}-w^{2}}$ ef $k=\frac{\left(\gamma \mu_{0}\right) M w}{\left(\gamma \mu_{0} H_{0}\right)^{2}-w^{2}}$

$\mu_{0}$ et $\mu_{\mathrm{r}}$ sont respectivement les perméabilités absolue et relative. $\gamma$ est la constante gyromagnétique $(\gamma=2.8 \mathrm{MHz} / 0 \mathrm{e})$. $W$ est la pulsation de travail. $M$ et $H_{0,}$ en oersteds, représentent respectivement l'aimantation et le champ magnétique statique appliqué au ferrite. 


\section{Lignes de transmission inhomogènes}

Les structures analysées sont constituées de plusieurs lignes planaires de transmission reposant sur des substrats hétérogènes et propageant le mode fondamental quasi-TEM. Ce mode est caractérisé par des composantes longitudinales nulles du champ électrique $E_{z}$, du champ magnétique $H_{z}$ et une constante de propagation $\beta$ dans la direction de propagation 0z. En supposant que les paramètres géométriques (dimensions) et électriques (permittivité, perméabilité) restent inchangés selon la direction de propagation (suivant la longueur de la ligne), la caractérisation d'un tel mode se ramène à la section transversale (Figure I) de la structure analysée en fonction des potentiels scalaire $v$ et vecteur $A$.

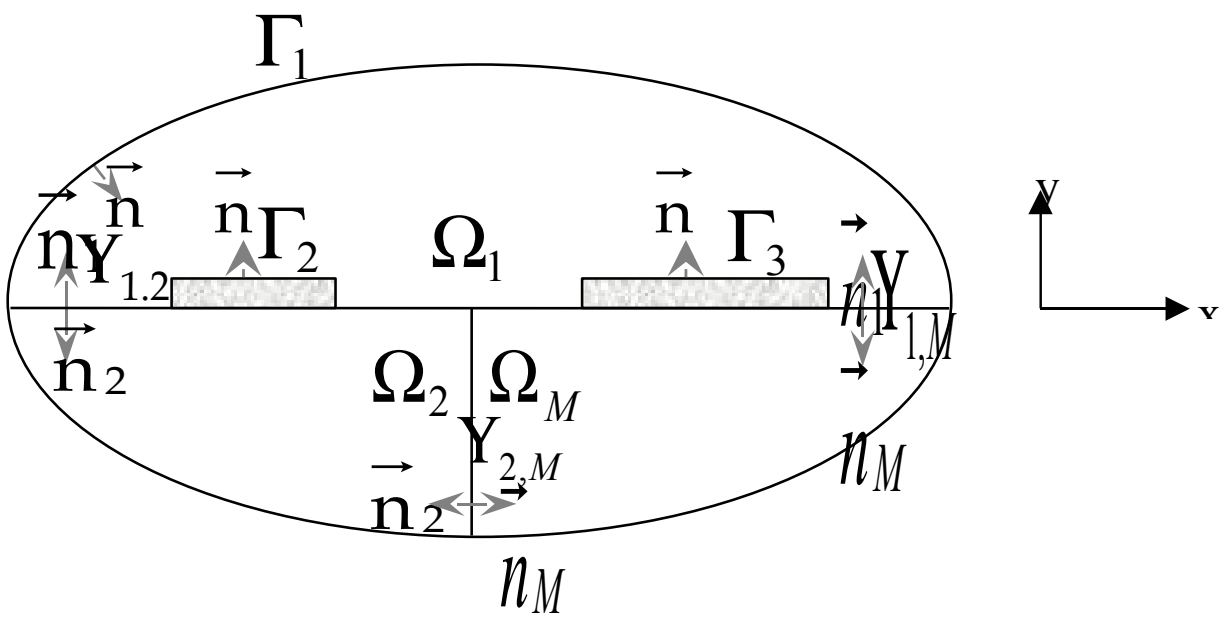

Figure 1 : Section transversale (plan $(x, y))$ d'une ligne de transmission inhomogène. $\Omega_{i}: i^{\grave{i} m e}$ domaine $\left(\mu_{r i}, \varepsilon_{r i}\right)$, i variant de $I$ à $M$ domaines (matériaux), $\Gamma_{1}$ : conducteur externe (blindage de la ligne) $\Gamma_{2}, \Gamma_{3}$ : rubans métalliques, $\vec{n}_{i}$ : normale à l'interface $\mathrm{Y}_{i, j}$ entre deux matériaux.

Les champs électriques $\vec{E}_{t}$ et magnétique $\vec{H}_{t}$ de cette onde quasi-TEM, décrits sur la section transversale (plan $\mathrm{t}=(\mathrm{x}, \mathrm{y})$ ), s'écrivent en fonction du potentiel scalaire $v$ et la composante longitudinale $A_{z}$ du potentiel vecteur, comme suit :

$$
\vec{E}_{t}=-\overrightarrow{\operatorname{grad}_{t}}(v)
$$

M. Meliani et al. 


$$
\vec{H}_{t}=\frac{1}{\mu_{0} \mu_{r}} \overrightarrow{\operatorname{grad}}_{t}\left(A_{z}\right) \wedge\left(\vec{u}_{z}\right)
$$

$\mu_{r}$ est le tenseur de perméabilité relative décrit par la relation (I) et $\vec{u}_{z}$ représente le vecteur unitaire de la direction de propagation $O z$.

En introduisant ce développement dans les équations de Maxwell, on obtient les équations fondamentales suivantes, vérifiés par les potentiels en tout point de chaque domaine $\Omega_{\mathrm{i}}, 1 \leq i \leq M$ :

$$
\begin{aligned}
& \operatorname{div}\left(\overrightarrow{\varepsilon . \operatorname{grad}_{t}}(v)\right)=0 \\
& \operatorname{div}\left(\frac{1}{\mu} \overrightarrow{\operatorname{grad}_{t}}\left(A_{z}\right)\right)=0
\end{aligned}
$$

Sur l'interface $Y_{i, j}$ de deux matériaux $i$ et $j, 1 \leq i \leq j \leq M$ (matériaux), les conditions de continuités s'écrivent :

$\varepsilon_{i} \overrightarrow{\operatorname{grad}}\left(v_{i}\right) \cdot \overrightarrow{n_{i}}+\varepsilon_{j} \overrightarrow{\operatorname{grad}}\left(v_{j}\right) \cdot \overrightarrow{n_{j}}=0 ; \quad \overrightarrow{\operatorname{grad}}\left(\mathrm{A}_{\mathrm{z}}\right)$ est continu.

Sur la surface $\Gamma_{\mathrm{p}} \mathrm{du}$ conducteur $\mathrm{p}, 1 \leq p \leq N_{c}$ (conducteurs) le potentiel $\boldsymbol{v}$ est constant et $A_{z}=\frac{1}{v_{p}} v$.

Sachant qu'il n'existe pas de solutions analytiques aux équations (5) et (6) décrivant des milieux de propagation complexes, on est alors amené à exploiter des méthodes numériques (méthode des éléments finis, méthode des différences finies, méthode des moments, ...) pour résoudre ce système. Dans cette étude, nous exposons les résultats d'analyse, par la méthode des éléments finis (MEF), des lignes de transmission planaires à plusieurs couches diélectrique -ferrite.

\section{Grandeurs caractéristiques du mode quasi-TEM}

La détermination des potentiels scalaire $v$ et vecteur $A_{z}$, permet la déduction des champs électromagnétiques transverses à partir des relations (3) et (4) et par la suite toutes les grandeurs caractéristiques de la structure de propagation. Ainsi, on cite :

M. Meliani et al. 
- L'énergie électrique moyenne $\bar{W}_{\mathrm{em}}$, emmagasinée dans la structure de propagation composée de M matériaux, est déduite du champ électrique transverse:

$$
\bar{W}_{e m}=\frac{1}{4} \sum_{i=1}^{M}\left(\iint \varepsilon_{0} \varepsilon_{r_{i}} \cdot \vec{E}_{t_{i}} \cdot \vec{E}_{t_{i}}^{*} d x d y\right)
$$

avec $E_{t_{i}}=E_{x} \vec{u}_{x}+E_{y} \vec{u}_{y}$; le champ électrique transverse dans le matériau i.

$\left(\vec{u}_{x}, \vec{u}_{y}\right)$ sont les vecteurs unitaires des directions ox et oy.

De même l'énergie magnétique moyenne $\bar{W}_{\mathrm{mm}}$ emmagasinée dans la structure, calculée à partir du champ magnétique transverse, est définie par :

$$
\bar{W}_{m m}=\frac{1}{4} \sum_{i=1}^{M}\left(\iint \mu_{0} \mu_{r_{i}} \vec{H}_{t_{i}} \vec{H}_{t_{i}}^{*} d x d y\right)
$$

- La capacité par unité de longueur entre deux conducteurs, portés aux potentiels $v_{1}$ et $v_{2}$, est exprimée par la relation :

$$
C=\frac{4 \bar{W}_{e m}}{\left|v_{1}-v_{2}\right|^{2}}
$$

Lorsqu'on étudie des lignes de transmission inhomogènes, on cherche toujours, afin de faciliter les calculs, à déduire une ligne fictive homogène équivalente, en remplaçant tous les matériaux par un seul de perméabilité relative équivalente $\mu_{\text {reff }}$ et de permittivité relative équivalente $\varepsilon_{\text {reff }}$. Ces paramètres sont définis par les relations suivantes:

$$
\begin{aligned}
& \varepsilon_{r e f f}=\varepsilon_{r e f f}^{\prime}-j \cdot \varepsilon_{r e f f}^{\prime \prime}=\frac{\iint \varepsilon_{0} \varepsilon_{r} \vec{E}_{t} \vec{E}_{t}^{*} \cdot d x \cdot d y}{\iint \varepsilon_{0} \vec{E}_{t v} \vec{E}_{t v}^{*} \cdot d x \cdot d y} \\
& \mu_{r e f f}=\mu_{r e f f}^{\prime}-j \cdot \mu_{r e f f}^{\prime \prime}=\frac{\iint \mu_{r v} \vec{H}_{t v} \vec{H}_{t v}^{*} \cdot d x \cdot d y}{\iint \mu_{r} \vec{H}_{t} \vec{H}_{t}^{*} \cdot d x \cdot d y}
\end{aligned}
$$


$\left(\mu_{r v}=\left[\begin{array}{ll}1 & 0 \\ 0 & 1\end{array}\right]\right)$ est le tenseur de perméabilité relative du vide .

$\vec{E}_{t v}$ et $\vec{H}_{t v}$ sont respectivement les champs électrique et magnétique dans la même structure remplie d'air (vide).

- La vitesse de phase $\mathcal{V}_{p}$ s'écrit :

$$
v_{p}=\frac{3.10^{8}}{\sqrt{\varepsilon_{r e f f} \mu}} \operatorname{en}(m / s)
$$

- La self par unité de longueur est directement déduite de la relation :

$$
L=\frac{1}{C v_{p}^{2}}
$$

- L'impédance caractéristique $Z_{c}$ de la ligne de transmission est ensuite déduite de la relation :

$$
Z_{c}=\frac{1}{v_{p} \cdot C}
$$

- La constante de propagation $\beta$ est déduite de la pulsation $\omega$ et de la vitesse de phase $v_{p}$ :

$$
\beta=\frac{\omega}{v_{p}}
$$

\section{Méthodologie d'analyse}

La méthode des éléments finis est une méthode numérique qui a fait preuve d'efficacité dans divers domaines (électromagnétisme, mécanique, ...). Les références $[6,7]$ développent la formulation de ce problème par la méthode des éléments finis, dont les principales étapes sont résumées dans les points suivants :

- La subdivision de la section droite (plan transverse $(x, y)$ ) de la ligne de transmission en un nombre fini de triangles. Dans chaque élément triangulaire, les propriétés du matériau (permittivité, perméabilité) sont constantes.

- Le potentiel scalaire $v$ ou le potentiel vecteur $A_{z}$, traduit par la fonction $G(x, y)$, est exprimé par la développement suivant :

$$
G(x, y)=\sum_{i=1}^{3} g_{i} \lambda_{i}(x, y)
$$

M. Meliani et al. 
$g_{i}$ représente la valeur nodale des potentiels sur le nœud i correspondant à un sommet d'un triangle. $\lambda_{i}(x, y)$ est un polynôme de Lagrange de degré 1 associé au nœud i.

- En introduisant ce développement dans les équations (5) et (6), et en intégrant sur le domaine d'étude, on obtient la forme variationnelle suivante du problème :

$$
\iint \operatorname{qgrad} G \cdot \operatorname{gra} d G d s=0
$$

$(S)$

Avec $q=\mathcal{E}$ pour $\mathrm{G}=v$ (potentiel scalaire) ou $q=\frac{1}{\bar{\mu}}$ pour $\mathrm{G}=A_{z}$.

L'introduction du développement polynomial de $G$ (relation (16)) dans (17) et l'intégration sur l'ensemble des éléments triangulaires du maillage conduit à un système numérique linéaire de la forme $[B]\{X\}=0$, à résoudre par des techniques numériques adéquates. La solution $\{X\}$ exprime les valeurs des potentiels en tous les sommets des triangles du maillage. La connaissance de ces grandeurs permet de déduire tous les paramètres essentiels (impédance caractéristique, vitesse de phase, constante de propagation, schéma du circuit électronique équivalent) de ces structures de propagation.

En utilisant cette approche, un logiciel [7] efficace a été développé, traduisant les étapes de maillage de la structure analysée, de prise en compte des conditions aux limites vérifiées par les potentiels sur les conducteurs, de résolution du système numérique linéaire et de calcul de toutes les grandeurs caractéristiques présentées auparavant. Afin de valider le modèle conçu, plusieurs structures de déphaseurs et de coupleurs agiles ont été analysées. Quelques résultats sont exposés dans les paragraphes suivants.

\section{Ligne micro ruban sur substrat diélectrique - ferrite}

Un déphaseur, constitué d'une simple ligne micro ruban reposant sur un substrat diélectrique-ferrite (figure 2), a été analysé en premier lieu.

Les paramètres électriques et géométriques du déphaseur sont décrits dans le Tableav 1. II est également supposé que le ruban métallique admet une épaisseur négligeable et que le champ magnétique statique de polarisation du ferrite est dirigé selon la direction de propagation $(0 z)$. 


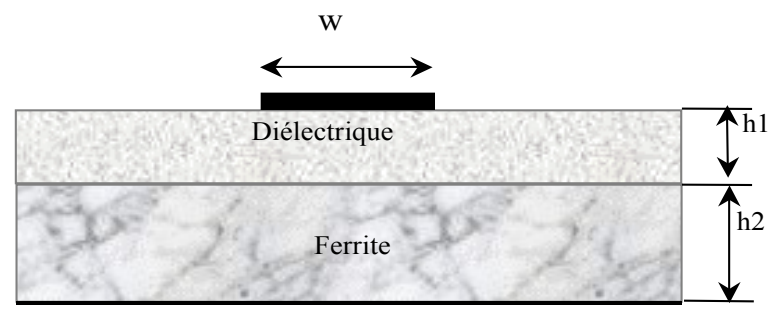

Figure 2. Ligne micro ruban sur substrat diélectrique $\left(\varepsilon_{r d}, \mu_{r}=1\right)$ et ferrite $\left(\varepsilon_{r f}, \mu_{r}\right)$.

Tableau 1 : Paramètres de la ligne micro ruban

\begin{tabular}{|c|c|c|c|c|c|}
\hline $\begin{array}{c}\mathrm{W} \\
(\mathrm{mm})\end{array}$ & $\begin{array}{c}\boldsymbol{h}_{1} \\
(\mathrm{~mm})\end{array}$ & $\begin{array}{c}\boldsymbol{h}_{2} \\
(\mathrm{~mm})\end{array}$ & $\boldsymbol{\varepsilon}_{r d}$ diél. & $\boldsymbol{\varepsilon}_{r f}$ ferr. & $\begin{array}{c}4 \pi M_{s} \\
(\text { Gauss })\end{array}$ \\
\hline 0.600 & 0.254 & 0.800 & 9.9 & 16.5 & 2800 \\
\hline
\end{tabular}

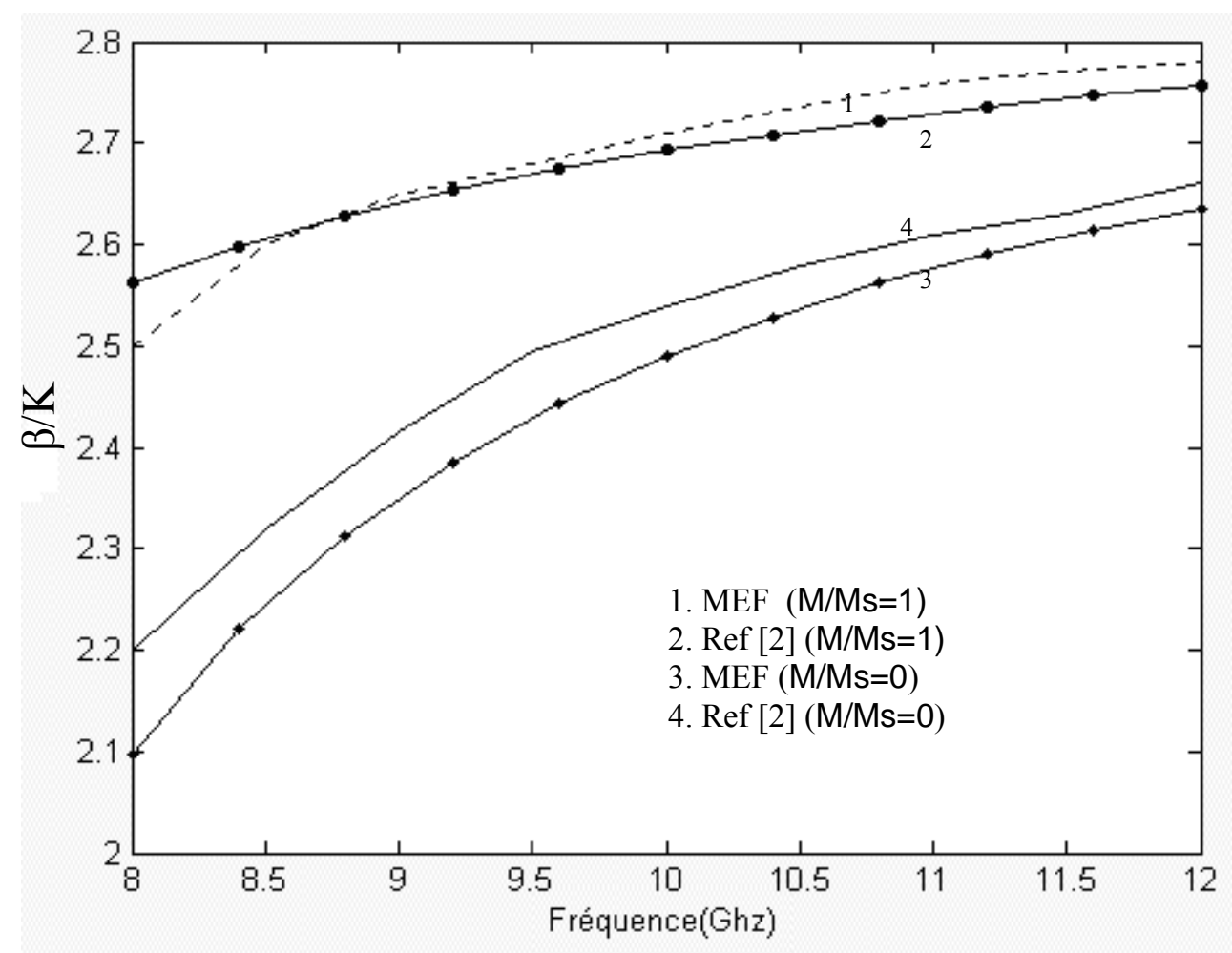

Figure 3 : Constante de phase normalisée $\left(\beta / K_{0}\right)$ en fonction de la fréquence pour un ferrite saturé $(M / M s=1)$ et pour un ferrite sans aimantation $(M / M s=0)$ 
L'analyse de ce déphaseur, a permis le calcul de la constante de propagation normalisée (ou constante de phase $\left(\beta / K_{0}\right)$ ) et de l'impédance caractéristique $Z_{c}$ du mode fondamental, dans la bande de fréquence [8-12] $\mathrm{GHz}$.

Les Figures 3 et 4 décrivent respectivement les variations, en fonction de la fréquence, de la constante de phase normalisée et de l'impédance caractéristique.

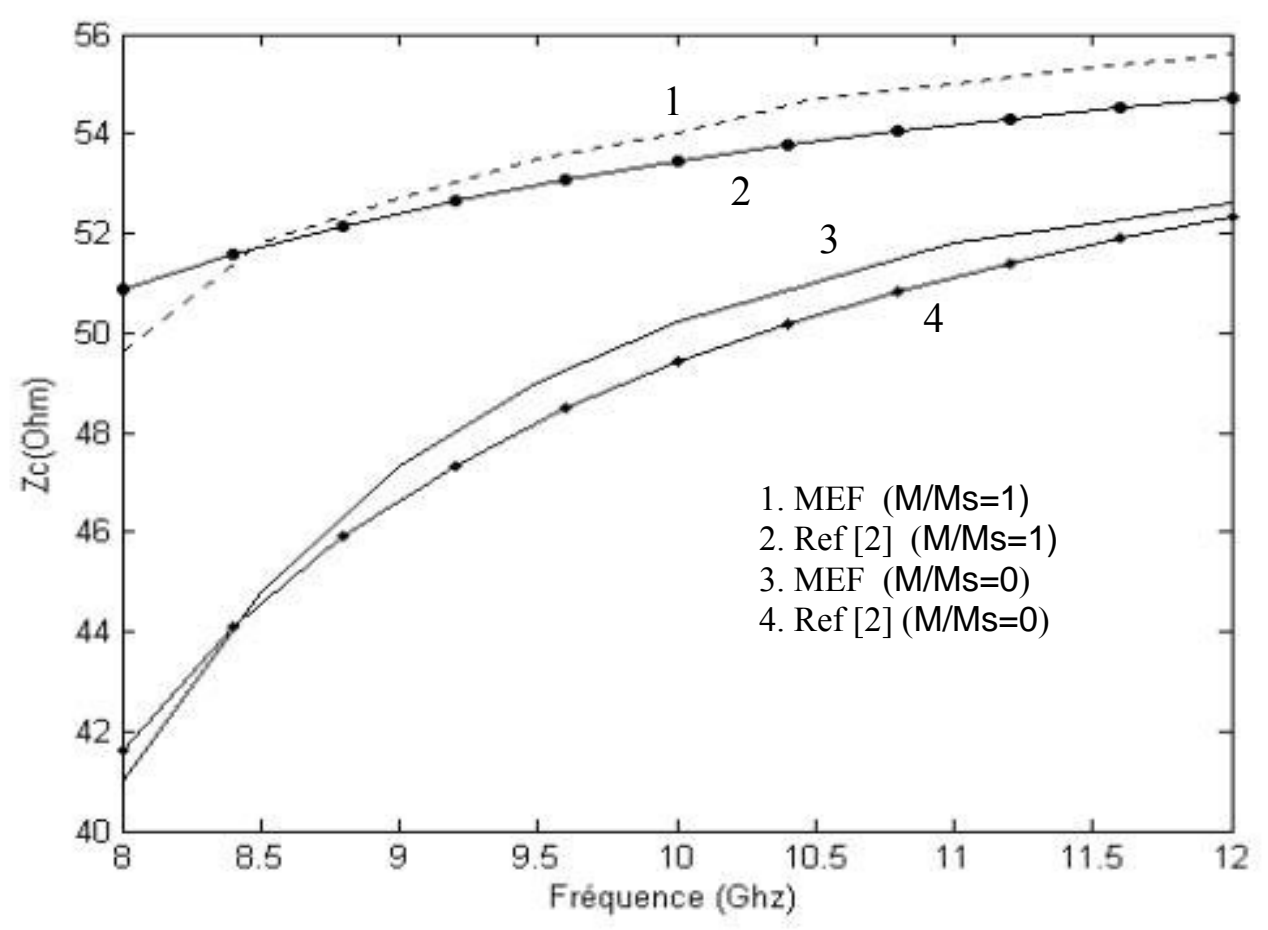

Figure 4 : Impédance caractéristique $Z_{c}$ en fonction de la fréquence pour un ferrite saturé $(M / M s=1)$ et pour un ferrite sans aimantation $(M / M s=0)$

Ces courbes montrent une bonne cohérence des résultats simulés avec ceux de la référence [2]; ce qui permet de valider le modèle numérique développé.

D'autre part, l'aimantation du ferrite par un champ magnétique statique (M/Ms non nul) engendre un déphasage ( $\beta \mid$ où I est la longueur de la ligne) et une impédance caractéristique plus importants par rapport à l'utilisation d'un ferrite isotrope (sans aimantation : $M / M s=0$ ). 


\section{Coupleur à ferrite}

La structure à étudier est constituée de deux lignes micro rubans symétriques, couplées et blindées, reposant sur un substrat en ferrite. La section transversale et les paramètres caractéristiques de ce coupleur sont décrits respectivement sur la Figure 5 et le Tableau 2.

a

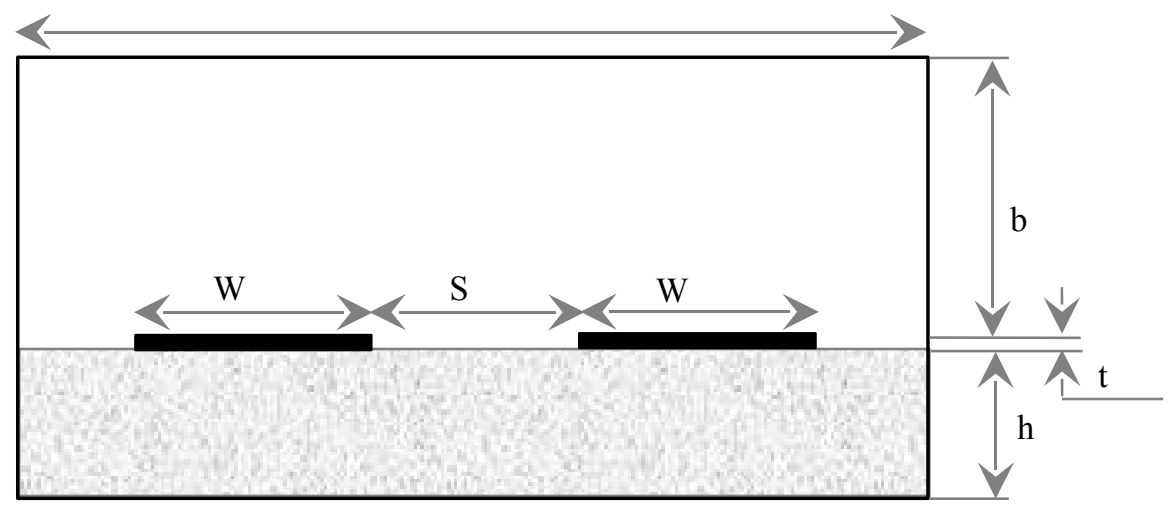

Figure 5. Lignes micro rubans, couplées et blindées, reposant sur un substrat à ferrite ( $\square)$.

Tableau 2. Paramètres du coupleur blindé à deux lignes micro rubans

\begin{tabular}{|c|c|c|c|c|c|c|}
\hline $\begin{array}{c}\mathrm{h} \\
(\mathrm{mm})\end{array}$ & $\begin{array}{c}\mathrm{b} \\
(\mathrm{mm})\end{array}$ & $\begin{array}{c}\mathrm{a} \\
(\mathrm{mm})\end{array}$ & $\begin{array}{c}\mathrm{W} \\
(\mathrm{mm})\end{array}$ & $\begin{array}{c}\mathrm{S} \\
(\mathrm{mm})\end{array}$ & $\begin{array}{c}\mathrm{t} \\
(\mathrm{mm})\end{array}$ & $\mathcal{E}_{\text {If }}$ \\
\hline 0.85 & 4.15 & 5.95 & 0.85 & 0.25 & 0.0254 & 9.0 \\
\hline
\end{tabular}

Le champ magnétique statique de polarisation du ferrite est orienté selon la direction de propagation $\left(\mathrm{O} z_{\mathrm{z}}\right.$. Les modes fondamentaux d'une telle structure sont des modes quasiTEM pair ou impair selon les potentiels appliqués aux deux rubans [1]. Dans telles conditions, on définit :

- Des impédances caractéristiques $Z_{c e}$ et $Z_{c o}$, calculées selon la relation (14), respectivement pour les modes pair et impair excités dans cette structure. 
- Un coefficient de couplage $k_{c}$, traduisant la qualité du coupleur, donné par :

$$
k_{c}=\frac{Z_{c e}-Z_{c o}}{Z_{c e}+Z_{c o}}
$$

L'influence du gap $S$ sur les impédances caractéristiques $Z_{c e}$ and $Z_{c o}$ des modes pairs et impairs est illustrée sur la Figure 6. Ces courbes montrent que les impédances caractéristiques des modes pairs sont plus élevées que celles des modes impairs pour les faibles valeurs du gap $S$, pour toute intensité de l'aimantation du ferrite.

Pour une aimantation donnée, ces impédances se rejoignent pour des valeurs élevées du gap S. De même, pour un gap donné, ces impédances augmentent avec l'intensité de l'aimantation du ferrite.

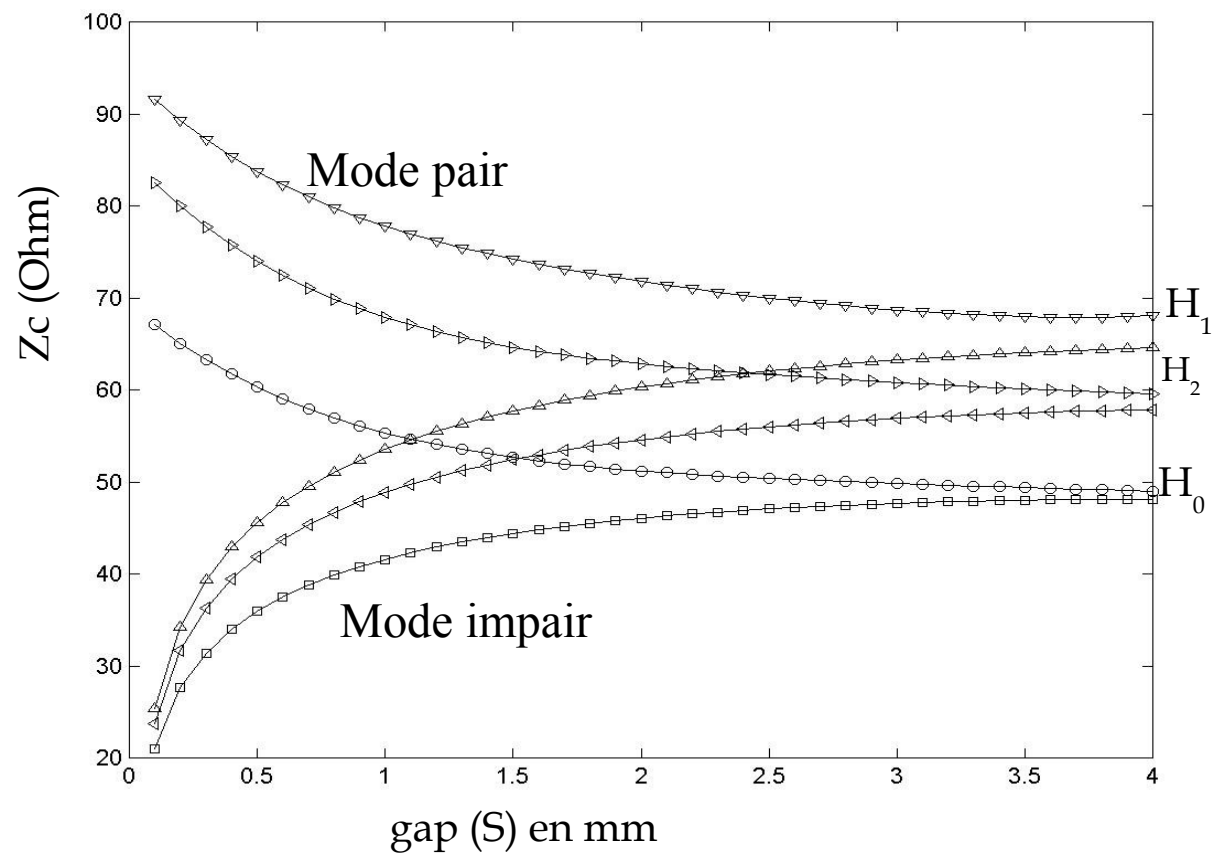

Figure 6. Impédance caractéristique en fonction du gap $S$ pour $\left(\mathrm{H1}=500 \mathrm{Oe}, \mathrm{H} 2=100 \mathrm{Oe}, \mathrm{H}_{0}=0\right)$

La Figure 7 montre l'influence du gap $S$ sur le coefficient de couplage $K_{c}$ pour différentes intensités de l'aimantation. Ce coefficient $K_{c}$ décrivant la qualité du coupleur, augmente avec l'intensité de l'aimantation du ferrite pour une valeur donnée du gap $S$.

Ces résultats démontrent bien qu'il est possible de modifier l'impédance caractéristique 
du coupleur et le coefficient de couplage entre les deux lignes micro rubans par simple action sur l'intensité de l'aimantation du ferrite.

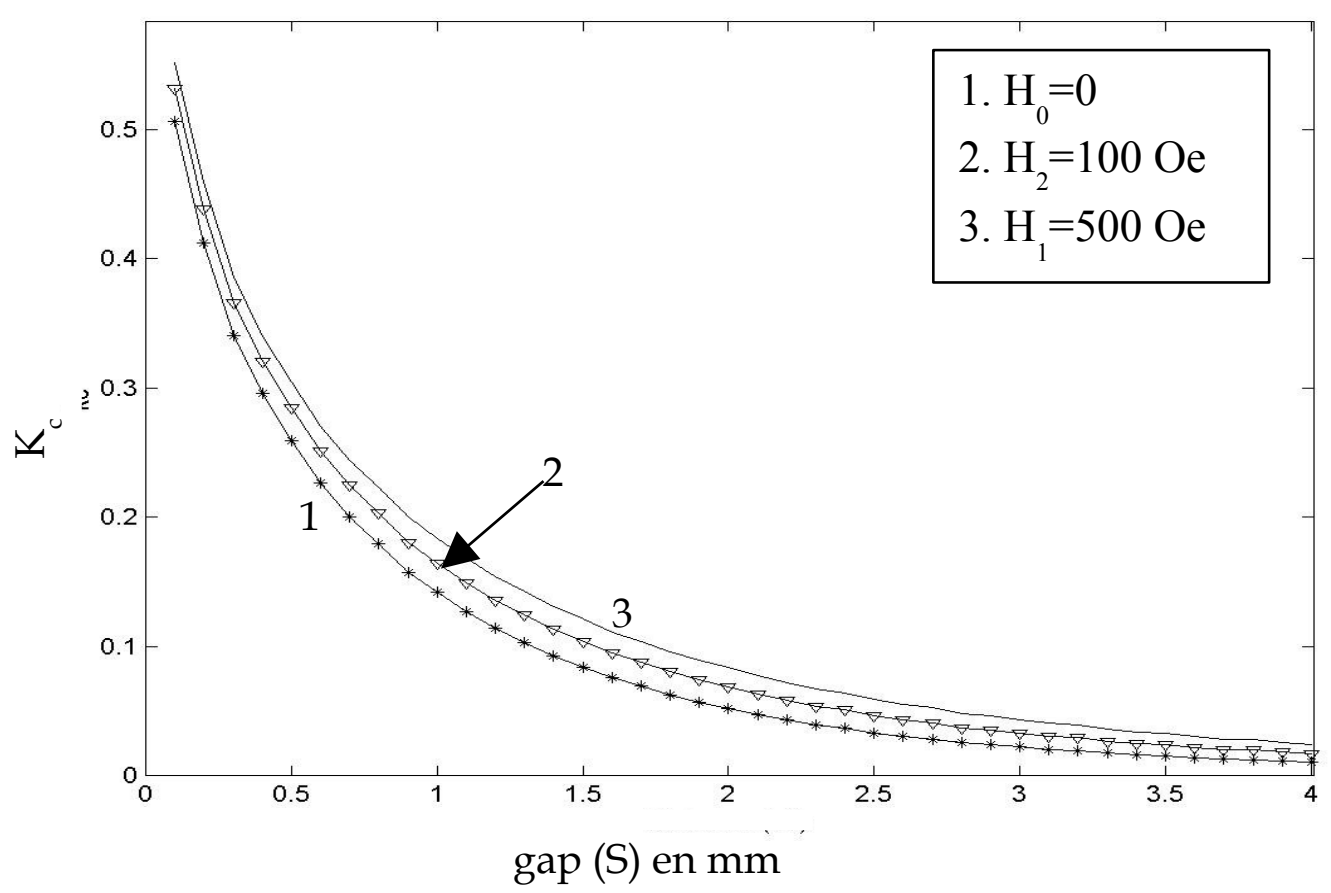

Figure 7 : Coefficient de couplage $K_{c}$ en fonction du gap $S$ $\operatorname{pour}\left(H_{l}=5000 e, H_{2}=1000 e, H_{0}=0\right)$

\section{Conclusion}

Les résultats simulés sont en accord avec ceux publiés ce qui démontre l'intérêt et l'efficacité du modèle numérique de CAO développé [7].

Ce logiciel assure d'une part l'analyse de l'influence des paramètres électriques et géométriques de la structure sous test sur ses grandeurs caractéristiques (constante de phase, impédance caractéristique, ...), d'autre part il permet la caractérisation de structures complexes et variées comportant plusieurs lignes de transmission placées sur des substrats à ferrite polarisé longitudinalement.

Cet outil numérique est actuellement exploité pour l'analyse des structures planaires à substrat en ferrite polarisé arbitrairement avec des intensités quelconques d'aimantation. 


\section{Références}

[1] - N. Benahmed, M. Feham, M. Kameche, "Finite element analysis of planar couplersII, Applied microwave and wireless, Vol. 12, № 10, 0ctober 2000

[2] - Y.C. Moon, J.R. Lee, S.W. Yun, and I.S. Chang, IIA broadband planar isolator using coupled microstrip lines on a magnetized gyrotropic substratell, Microwave Journal, Vol. 44, No 11, november 2001

[3] - P. Gelin and K. Berthou-Pichavant, IINew consistent model for ferrite pemeability tensor with arbitrary magnetization statell, IEEE Transaction on Microwave theory and techniques. Vol. 45, № 8, August 1997

[4] - S. Mallegol, P. Queffelec and M. Le Floch. "ICaractérisation large bande de matériaux ferrimagnétiques dans un état quelconque d'aimantationll, $7^{\text {ème }}$ Journées de microondes et materiaux, Toulouse-France. JCMM 2002

[5] - P. T. Theo, J. A. Jose, Y. B. Gan and V. K. Varadan, IAdaptative ferroelectric phased shifters for phased array antennall, IEEE on MTT, Center for the engineering of electronic and acoustic materials, the Pennsylvania State University, Singapore 118230, 1996

[6] - L. Zhou and L. E. Davis, "Finite element method with edge elements for waveguides loaded with ferrite magnetized in arbitrary directionnl, IEEE Transactions on MTT. Vol. 44, № 6, June 1996

[7] - B. Benbakhti, "IAnalyse et conception des dispositifs microélectroniques d̀ ferritesı, Thèse de Magister, Université de Tlemcen, Algérie, Juin 2002

M. Meliani et al. 